\title{
Emergency department ultrasound for the detection of B-lines in the early diagnosis of acute decompensated heart failure: a systematic review and meta-analysis
}

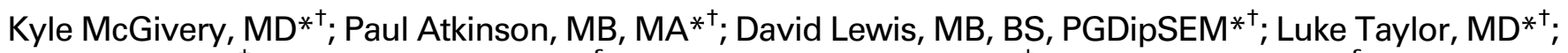 \\ Tim Harris, $\mathrm{MB}^{\ddagger}$; Kathleen Gadd, MLIS ${ }^{\S}$; Jacqueline Fraser, RNBN* ${ }^{+}$; George Stoica, $\mathrm{PhD}^{\S}$
}

\section{CLINICIAN'S CAPSULE}

What is known about the topic?

Current diagnostic tools for heart failure in the emergency department (ED) have limited accuracy and often lead to delays in management.

What did this study ask?

What is the accuracy of early bedside lung ultrasound in the diagnosis of acute decompensated heart failure?

What did this study find?

This meta-analysis found that the sensitivity and specificity of bedside lung US in ADHF is $82.5 \%$ and $83.6 \%$, respectively.

Why does this study matter to clinicians?

The implementation of early bedside lung US in the ED may lead to more accurate and timely diagnoses of ADHF.

\section{ABSTRACT}

Objectives: Dyspnea is a common presenting problem that creates a diagnostic challenge for physicians in the emergency department (ED). While the differential diagnosis is broad, acute decompensated heart failure (ADHF) is a frequent cause that can be challenging to differentiate from other etiologies. Recent studies have suggested a potential diagnostic role for emergency lung ultrasound (US). The objective of this systematic review was to assess the accuracy of early bedside lung US in patients presenting to the ED with dyspnea.

Methods: A systematic search of EMBASE, PubMed, and the Cochrane Library was performed in addition to a grey literature search. We selected prospective studies that reported on the sensitivity and specificity of B-lines from early lung ultrasound in dyspneic patients presenting to the
ED. Selected studies underwent quality assessment using the Critical Appraisal and Skills Program (CASP) questionnaire.

Data Extraction and Synthesis: The search yielded 3674 articles; seven studies met inclusion criteria and fulfilled CASP requirements for a total of 1861 patients. Summary statistics from the meta-analysis showed that as a diagnostic test for ADHF, bedside lung US had a pooled sensitivity of $82.5 \%(95 \%$ confidence interval $[\mathrm{Cl}]=66.4 \%$ to $91.8 \%)$ and a pooled specificity of $83.6 \%$ ( $95 \% \mathrm{Cl}=72.4 \%$ to $90.8 \%$ ).

Conclusions: Our results suggest that in patients presenting to the ED with undifferentiated dyspnea, B-lines from early bedside lung US may be reliably used as an adjunct to current diagnostic methods. The incorporation of lung US may lead to more appropriate and timely diagnosis of patients with undifferentiated ADHF.

\section{RÉSUMÉ}

Objectif: La dyspnée est un problème fréquent, difficile à diagnostiquer correctement par les médecins au service des urgences (SU). Certes, il existe un large éventail de diagnostics différentiels, mais l'insuffisance cardiaque décompensée aiguë (ICDA) est une cause fréquente de ce trouble, mais elle peut être difficile à distinguer d'autres causes. D'après des études récentes, l'échographie des poumons au SU pourrait jouer un rôle dans la pose du diagnostic. La revue systématique dont il sera question ici visait à évaluer l'exactitude de l'échographie précoce des poumons au chevet chez les patients souffrant de dyspnée au SU.

Méthode: La recherche consistait en une revue systématique des bases de données EMBASE, PubMed et Cochrane Library, de même qu'en un examen de la documentation parallèle. Nous avons procédé à une sélection des études prospectives qui faisaient état de la sensibilité et de la spécificité des lignes B observées à l'échographie précoce des poumons chez les patients souffrant de dyspnée au SU. Les études sélectionnées

From the * Department of Emergency Medicine, Dalhousie University, Saint John, NB; †Saint John Regional Hospital Department of Emergency Medicine, Saint John, NB; ¥The London Hospital, London, United Kingdom; and §Horizon Health Network, Saint John, NB.

Correspondence to: Dr. Kyle McGivery, Saint John Regional Hospital, Saint John, NB E2L 4L2, Canada; Email: kyle.mcgivery@dal.ca 
ont été soumises au questionnaire Critical Appraisal and Skills Program (CASP) en vue de l'évaluation de la qualité.

Extraction des données et synthèse: La recherche a permis de relever 3674 articles, dont 7 respectaient les critères de sélection ainsi que les exigences du questionnaire CASP, ce qui a porté à 1861 le nombre total de patients. Des statistiques sommaires provenant de la méta-analyse a révélé que l'échographie des poumons au chevet, en tant qu'examen de diagnostic de I'ICDA, avait une sensibilité globale de $82,5 \%$ (intervalle de confiance [IC] à $95 \%: 66,4 \%$ à $91,8 \%$ ) et une spécificité globale de 83,6 \% (IC à $95 \%$ : 72,4 \% à 90,8 \%).
Conclusions: Les résultats de l'étude donnent à penser que l'échographie précoce des poumons en vue de la détection de lignes B chez les patients souffrant de dyspnée d'origine indéterminée au SU pourrait compléter, de manière fiable, les méthodes actuelles de diagnostic. L'intégration de l'échographie des poumons au SU pourrait donc faciliter et accélérer la pose du diagnostic d'ICDA chez les patients souffrant de dyspnée d'origine indéterminée.

Keywords: lung ultrasound, heart failure, B-lines, dyspnea, emergency department

\section{INTRODUCTION}

Dyspnea is a frequent presenting problem in emergency department (ED) patients that requires a broad differential diagnosis. It creates a challenge for emergency physicians, often forcing them to make a rapid diagnosis and initiate treatment with limited clinical information. ${ }^{1}$ While there are many potential underlying causes, acute decompensated heart failure (ADHF) is among the most common and challenging etiologies to be considered. ${ }^{2}$ Recent statistics suggest that the individual lifetime risk of developing heart failure in North America is one in five, with prevalence increasing with age. ${ }^{3}$ With a population that continues to age, a $25 \%$ increase in heart failure prevalence is estimated by the year 2030. ${ }^{4}$ Of those patients presenting to the ED with $\mathrm{ADHF}$, over $80 \%$ are admitted to the hospital, making it the most common reason for admission and a significant financial burden on the health care system. ${ }^{4}$

Despite the prevalence of heart failure, the standard workup for dyspneic patients in the ED is non-specific and often fails to differentiate ADHF from conditions such as chronic obstructive pulmonary disease (COPD). ${ }^{5,6}$ This distinction is, however, essential as mismanagement has been shown to affect the morbidity and mortality of dyspneic patients negatively. ${ }^{7,8}$ In addition to a history and physical exam, diagnostic modalities for dyspnea often include a chest $\mathrm{x}$-ray (CXR), brain natriuretic peptide (BNP), and, less commonly, a computed tomography (CT) scan. ${ }^{9}$ CXR findings such as vascular redistribution, cardiomegaly, and interstitial edema have been shown to be highly subjective; inter-rater agreement between radiologists and emergency physicians has been estimated to be as low as 50\%. ${ }^{10-12}$ While BNP and N-terminal probrain-type natriuretic peptide (NT-ProBNP) may help in differentiating dyspneic patients, these assays are often elevated in non-ADHF disease processes such as acute coronary syndromes, right heart strain, renal failure, and advanced age. ${ }^{13}$ Alternatively, BNP may be falsely low in patients with a high body mass index (BMI), adding to the diagnostic confusion. ${ }^{1}$ Overall, approximately $20 \%$ of patients presenting to the ED with dyspnea are misdiagnosed and treated inappropriately. ${ }^{1,12}$

Point-of-care ultrasound (US) is an emerging and increasingly helpful clinical adjunct for the rapid bedside diagnosis of many conditions in the ED. Evidence currently supports its use in trauma, gynecological, procedural, cardiac, and vascular presentations. ${ }^{14}$ Several recent studies have also suggested a potential role for bedside lung US in diagnosing ADHF. ${ }^{5}$ Unlike many US applications, lung US is largely based on artifacts as sounds waves scatter while passing through the air. A-lines are horizontal reverberation lines that may be present in patients with normal lungs, asthma, or COPD. B-lines are vertical artifacts that represent fluid-filled alveoli or interstitial edema that can be caused by pneumonia, pulmonary fibrosis, acute respiratory distress syndrome (ARDS), and ADHF. ${ }^{15}$ In conjunction with the clinical presentation, B-lines present in multiple lung areas bilaterally are highly suggestive of ADHF. Several protocols exist for the diagnosis of ADHF using B-lines, the most common being the Volpicelli method that requires three or more B-lines in two or more lung zones bilaterally. ${ }^{2,16}$ This method of bedside lung US provides rapid diagnostic information that may allow for earlier and more appropriate treatment of dyspneic patients.

Although numerous studies have suggested that B-lines can help in diagnosing $\mathrm{ADHF}$ reliably in undifferentiated dyspneic patients, ${ }^{11,12,17}$ many of these 
studies have used small sample sizes and have often combined lung US with echocardiography and an inferior vena cava (IVC) measurement. A 2015 metaanalysis by $\mathrm{Al}$ Deeb et al. found that the sensitivity and specificity of B-lines for the diagnosis of ADHF was $94 \%$ and $92 \%$, respectively. ${ }^{6}$ However, this analysis included studies performed in intensive care units (ICU), internal medicine wards, and prehospital settings, in addition to EDs. Martindale et al. performed a more recent meta-analysis that included prehospital patients and reviewed several diagnostic modalities including lung US, electrocardiogram, CXR, BNP, echocardiography, and bioimpedance. ${ }^{18}$ To our knowledge, no study has systematically reviewed the literature on early lung US for B-lines alone in undifferentiated dyspneic patients in the ED.

The clinical question addressed in this systematic review was the following: for patients presenting to the ED with undifferentiated dyspnea, can B-line patterns in early bedside lung ultrasound be used to confirm a diagnosis of ADHF accurately?

\section{METHODS}

\section{Study design}

A systematic review protocol was created specifically to address the question and was agreed upon by all co-investigators a priori (see Data Supplement S1). The preferred reporting items for systematic reviews and meta-analyses (PRISMA) statement ${ }^{19}$ was followed for this systematic review.

\section{Search strategy}

With the assistance of a medical librarian, we conducted a systematic search of the following databases: MEDLINE (via PubMed), EMBASE, and the Cochrane Library. Studies were not limited based on language, type, or year of publication. Appropriate search terms were found, selected, and agreed upon by KM and KG, and a draft of the search strategy was circulated to the co-investigators for review. The search strategy was tested to ensure that it retrieved relevant hand-searched articles. The search strategy was also submitted to the Peer Reviewed Electronic Search Strategies (PRESS) forum for review by other health sciences librarians. The systematic search was completed on 2 August 2016. The grey literature search included hand-searching the reference lists for potentially eligible articles and previous reviews. The previous ten years of conference abstracts from selected major conferences were searched, and experts in the field were contacted to inquire about new, relevant literature. Exact duplicates were removed by the duplicates function of RefWorks (ProQuest, LLC, Ann Arbor, MI). Close duplicates were examined individually and only deleted if they were suspected of representing the same data source. The remaining unique titles were then uploaded to Covidence (Veritas Health Innovation, Victoria, Australia) for title and abstract screening.

\section{Study selection}

Prior to assessing articles found during our literature search, we developed strict inclusion and exclusion criteria to methodologically screen studies. We included prospective studies that reported on the sensitivity and specificity of B-lines in dyspneic ED patients. The reference standard in each study was a chart review for the diagnosis of $\mathrm{ADHF}$ based on all assessments and diagnostic results excluding lung US. Two reviewers (KM and LT) independently selected papers relevant to the research topic based on the title and abstract review of the search results. To ensure that all relevant articles were reviewed, any study in which a lung US was performed on dyspneic patients in the ED was included for a full-text review. If there was uncertainty about the relevance of a study during the abstract screening, it was automatically included for full-text scoring. Disagreements were resolved by consensus between reviewers. Two separate reviewers (DL and PA), who have significant experience in bedside US, reviewed the full-text articles using the inclusion criteria to determine which studies to include in the final analysis. Consensus was reached based on a discussion as to which articles should be included without the need for an arbitrator. An additional focused search of the literature for the intervening period between the completion of the manuscript and submission for publication was performed to ensure that recent articles were not missed.

\section{Quality assessment}

Each paper selected during the full-text review underwent an independent quality assessment by two reviewers (DL and PA) using a modified version of the Critical Appraisal Skills Program (CASP) checklist. $^{20}$ 
The CASP questionnaire is a diagnostic test checklist that helps in the assessment of the validity, bias, and applicability of diagnostic studies. This tool was chosen because it has been developed specifically for the critical appraisal of diagnostic test studies. To be considered for inclusion, each study had to meet all criteria in the CASP checklist. Disagreement in the CASP scores was dealt with by consensus between reviewers on a case-by-case basis.

\section{Data analysis}

Statistical analysis was performed using the Open Meta-Analyst software (Open Meta-Analyst, Brown University, Providence, RI) to calculate pooled sensitivities and specificities with a $95 \%$ confidence interval (CI) and summary receiver operating characteristic (SROC) curve. Likelihood ratios were then calculated based on this model. Measures of heterogeneity were also calculated and reported using the $I^{2}$ statistic. The pooled analysis was conducted using a random effects model.

\section{RESULTS}

\section{Study selection}

Our systematic electronic search yielded 3655 unique titles after any duplicates were removed. We identified an additional 19 studies through our grey literature search for a total of 3674 unique titles (Figure 1). While assessing the literature surrounding our clinical question before conducting the formal search, we had identified six articles by hand. These six papers were cross-referenced with our electronic search, and all had been included. Of the 3674 papers, 3633 were excluded based on the title and abstract screening process. After reviewing the full text of 41 articles, 34 studies did not meet our inclusion criteria for various reasons as described in Figure 1. If it was unclear whether the studies met the inclusion criteria, the authors were contacted on an individual basis. We selected seven articles,10,12,17,21-23 (Table 1) for inclusion in the meta-analysis that provided a sample of 1861 patients. All of these 1861 patients underwent bedside lung US in the ED for undifferentiated dyspnea. Subsequently, they each had a final clinical diagnosis that was blinded from the US results and served as the reference standard.

\section{Study characteristics}

In line with the selection criteria, all studies performed a bedside lung ultrasound in the ED. The included studies were completed in EDs of variable sizes (rural, urban, and academic) in four countries: three in the United States, ${ }^{1,10,23}$ two in Italy, ${ }^{12,21}$ one in Japan, ${ }^{22}$ and one in France ${ }^{17}$ (Table 1). Three studies ${ }^{1,10,23}$ reported either multiple protocols or results from each lung zone independently. In these instances, the data that reflected the Volpicelli method were used for the analysis (i.e., three or more B-lines in two or more lung zones bilaterally). One study ${ }^{22}$ used a modified Volpicelli method in which a positive lung zone was defined as any number of B-lines. All included studies used at least one of the following alternate tests in their clinical diagnosis: CXR, BNP, NT-proBNP, or echocardiography. Importantly, data from these tests were blinded from the sonographers. The bedside US was performed by emergency physicians, emergency medicine residents, ultrasound fellows, medical students, and cardiologists. Studies that used measures reported by either residents or medical students had inter-rater reliability ranging from $82 \%$ to $92 \%$ (Table 1 ).

\section{Quality assessment}

All studies meeting the inclusion criteria also met the requirements for methodological quality using the CASP questionnaire (Data Supplement S2). To be considered high quality, studies had to fulfill all CASP domains that include: blinding of the results, use of an appropriate reference standard, and reporting of the specificity and sensitivity of the B-lines. For the latter, studies were included if data were either available from the author or through extrapolation of presented data. Risk of bias was considered low in all studies that met the inclusion criteria, and no studies were excluded based on a quality assessment.

\section{Meta-analysis}

The random effects pooled results for sensitivity and specificity for ED-performed bedside lung US for the diagnosis of $\mathrm{ADHF}$ were $82.5 \%$ (95\% CI 66.4\%-91.8\%) and $83.6 \%$ (95\% CI 72.4\%-90.8\%), respectively (Figure 2). Figure 3 shows the hierarchical summary receiver operating characteristic (HSROC) curve for the meta-analysis. The positive likelihood ratio was 4.840 (95\% CI 2.57-9.09), 


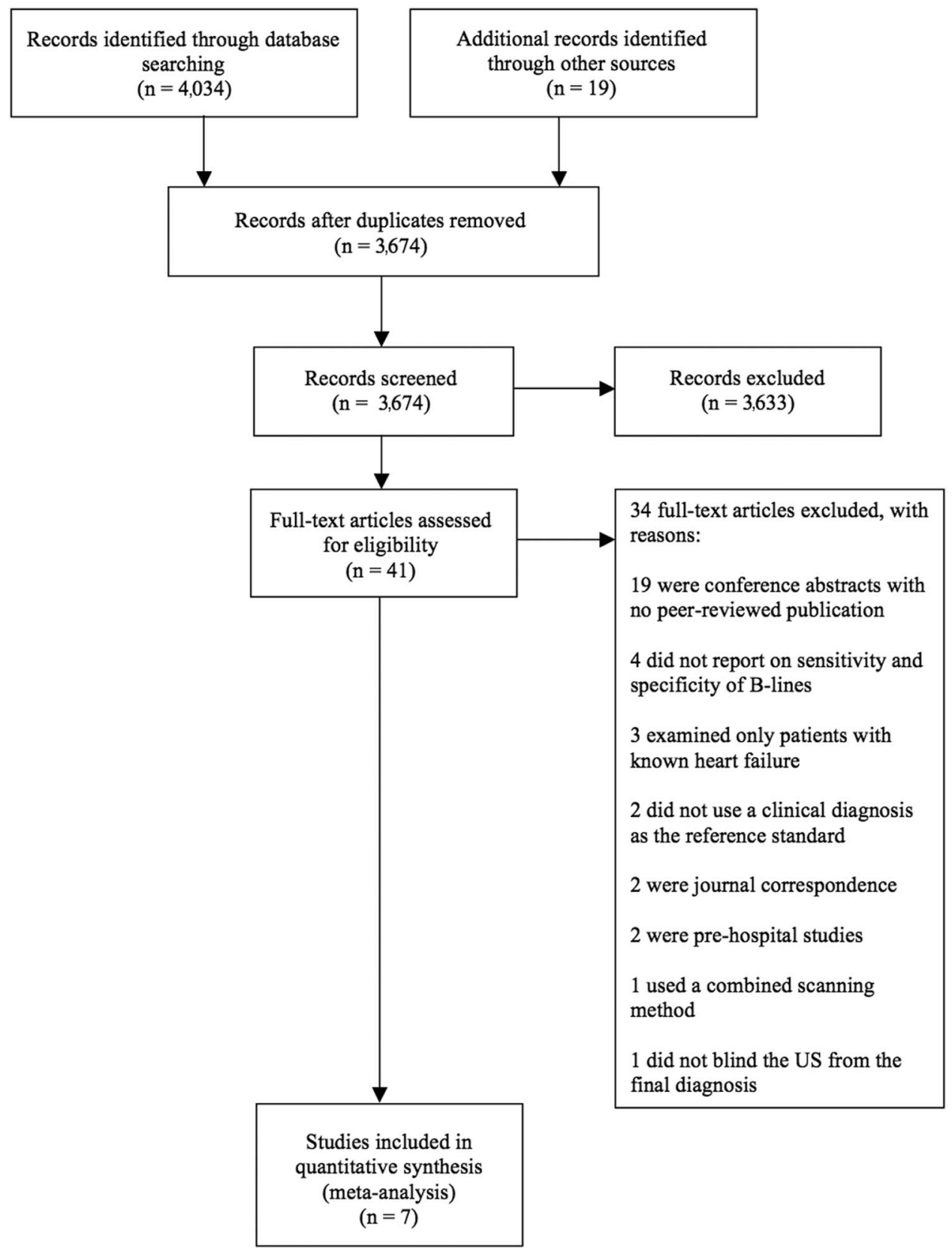

Figure 1. PRISMA flow diagram

and the negative likelihood ratio was 0.189 (95\% CI 0.09-0.39). The diagnostic odds ratio was 25.68 (95\% CI 7.34-89.88), and there was significant heterogeneity $\left(I^{2}=93.72\right)$. A subgroup analysis suggested that the sample sizes contributed significantly to the heterogeneity measure. Given this level of heterogeneity, a second meta-analysis including physician-only sonographer studies (excluded studies involving medical students and residents) was conducted. This analysis included five studies $^{12,17,21-23}$ and had sensitivity and specificity estimates of $88.6 \%$ (95\% CI 79.6\%-94.0\%) and $83.2 \%$ (95\% CI 63.2\%-93.5\%), respectively. The $I^{2}$ estimate for heterogeneity in the physician-only analysis was 77.2.

\section{DISCUSSION}

The purpose of this meta-analysis was to assess the accuracy of B-lines in ED-performed lung US examinations for the diagnosis of ADHF. Through our systematic review protocol, we identified seven studies, 


\begin{tabular}{|c|c|c|c|c|c|c|}
\hline Study & Setting & $N$ & Alternate test & Criterion standard & Sonographer & Methodology \\
\hline $\begin{array}{l}\text { Anderson } \\
\text { et al. }^{23}\end{array}$ & $\begin{array}{l}\text { USA; Academic } \\
\text { ED }\end{array}$ & 101 & CXR, BNP & $\begin{array}{l}\text { Medical record review by two } \\
\text { emergency physicians } \\
\text { ( } k=0.97 ; 95 \% \mathrm{Cl} 0.94-0.99 \text { ); } \\
\text { blinded to US data }\end{array}$ & $\begin{array}{l}\text { Emergency physicians; } \\
\text { emergency US fellows }\end{array}$ & $\begin{array}{l}\text { Reported both the Volpicelli method and } \\
\text { total B-line count. Reported data on } \\
\text { B-lines combined with } \\
\text { echocardiography; separate IVC } \\
\text { measurement }\end{array}$ \\
\hline Chiem et al. ${ }^{10}$ & $\begin{array}{l}\text { USA; Academic } \\
\text { ED }\end{array}$ & 380 & CXR & $\begin{array}{l}\text { Medical record review by one } \\
\text { emergency physician and one } \\
\text { cardiologist blinded to US data }\end{array}$ & $\begin{array}{l}\text { Emergency physicians, } \\
\text { residents }\end{array}$ & $\begin{array}{l}\text { Reported each lung zone } \\
\text { independently. Resident scans } \\
\text { interpreted at bedside by physician } \\
\text { (92\% interrater agreement); US } \\
\text { trained in two-week rotation and } \\
\text { formal teaching session on lung US }\end{array}$ \\
\hline Cibinel et al. ${ }^{12}$ & Italy; ED & 56 & CXR, echo & $\begin{array}{l}\text { Medical record review by one } \\
\text { emergency physician and one } \\
\text { cardiologist blinded to US data }\end{array}$ & Emergency physicians & $\begin{array}{l}\text { Volpicelli method; all patients had echo } \\
\text { before final diagnosis; median door-to- } \\
\text { ultrasound time was } 30 \text { minutes; } \\
92.2 \% \text { inter-rater agreement between } \\
\text { expert and inexperienced } \\
\text { sonographers }\end{array}$ \\
\hline $\begin{array}{l}\text { Kajimoto } \\
\text { et al. }^{22}\end{array}$ & Japan; ED & 90 & $\begin{array}{l}\text { CXR, BNP, bedside } \\
\text { echo }\end{array}$ & $\begin{array}{l}\text { Medical record review by one } \\
\text { cardiologist and two } \\
\text { pneumonologists blinded to US } \\
\text { data }\end{array}$ & Cardiologist & $\begin{array}{l}\text { Modified Volpicelli method (any B lines } \\
\text { in two or more zones bilaterally); lung } \\
\text { US completed within one minute and } \\
\text { prior to IVC and cardiac scanning. All } \\
\text { scans completed within } 30 \text { minutes of } \\
\text { arrival to ED }\end{array}$ \\
\hline Liteplo et al. ${ }^{1}$ & $\begin{array}{l}\text { USA; academic } \\
\text { ED }\end{array}$ & 94 & NT-proBNP & $\begin{array}{l}\text { Medical record review by two } \\
\text { emergency physicians } \\
\text { ( } \mathrm{k}=0.87 ; 95 \% \mathrm{Cl} 0.76-0.97 \text { ) } \\
\text { blinded to US data }\end{array}$ & $\begin{array}{l}\text { Emergency physicians; } \\
\text { medical students }\end{array}$ & $\begin{array}{l}\text { Reported Volpicelli method and data on } \\
\text { multiple lung zone combinations; US } \\
\text { performed by medical student and EP; } \\
82 \% \text { inter-rater agreement; median } \\
\text { time to US = } 79 \text { minutes }\end{array}$ \\
\hline Pivetta et al. ${ }^{21}$ & $\begin{array}{l}\text { Italy; multi- } \\
\text { centred: } \\
\text { seven EDs }\end{array}$ & 1005 & $\mathrm{CXR}, \pm \mathrm{BNP}$ & $\begin{array}{l}\text { Medical record review by one } \\
\text { emergency physician and one } \\
\text { cardiologist ( } \mathrm{k}=0.93 ; 95 \% \mathrm{Cl} \\
0.91-0.95 \text { ) blinded to US data }\end{array}$ & Emergency physicians & $\begin{array}{l}\text { Volpicelli method; physician provided } \\
\text { differential diagnosis before and after } \\
\text { lung US; all US performed within one } \\
\text { hour of ED presentation }\end{array}$ \\
\hline
\end{tabular}




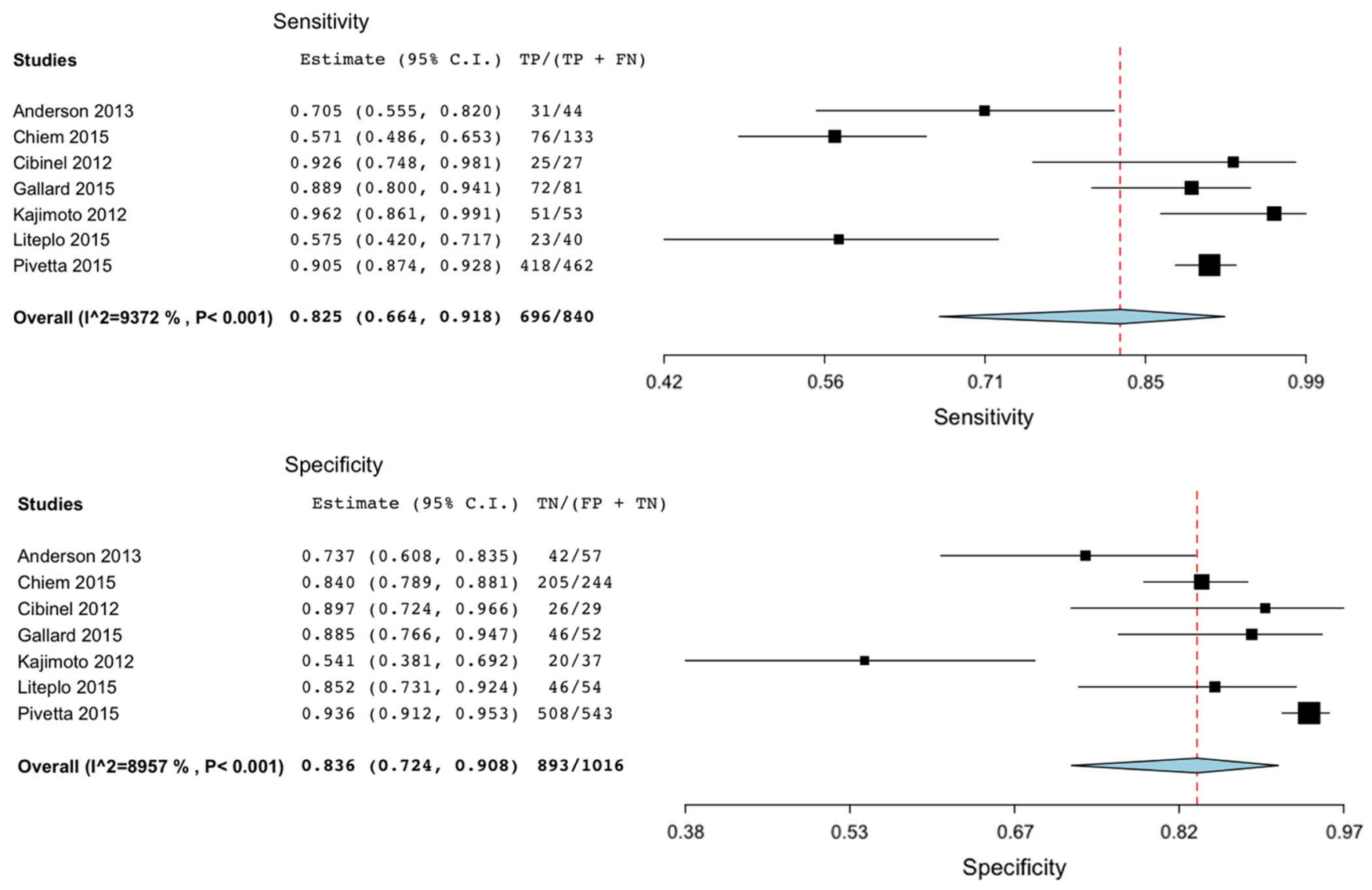

Figure 2. Summary and forest plot of individual and pooled sensitivity and specificity.

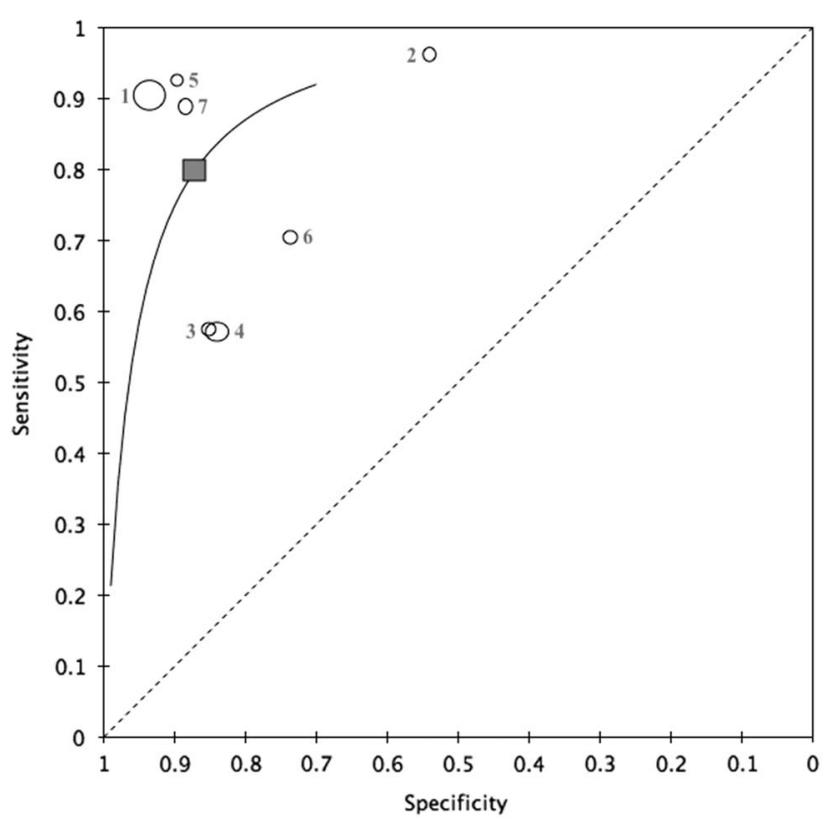

Figure 3. HSROC curve of the primary meta-analysis. $1=$ Pivetta et al. $2015 ; 2=$ Kajimoto et al. $2012 ; 3=$ Liteplo et al. 2009; $4=$ Chiem et al. 2015; $5=$ Cibinel et al. 2012; $6=$ Anderson et al. 2013; 7= Gallard et al. 2015. all of which satisfied the CASP requirements during a quality assessment. No studies were excluded based on the methodological quality which was likely because of the selective inclusion criteria. The pooled sensitivity and specificity of $82.5 \%(95 \%$ CI $66.4 \%-91.8 \%)$ and $83.6 \%$ (95\% CI $72.4 \%-90.8 \%$ ), respectively, suggest that bedside lung US offers a reliable diagnostic adjunct for the early differentiation of dyspneic patients. Previously, the sensitivity and specificity of CXR for the identification of ADHF have been estimated to be $57 \%$ and $78 \%$, respectively. ${ }^{24}$ BNP can be helpful, but often results in false positives, and may create ambiguity concerning choosing threshold values. ${ }^{25,26}$ More importantly, results from these conventional tests are often delayed, and lung US offers real-time information. Not all studies reported the time required to perform a lung US examination; however, Kajimoto et al. $^{22}$ performed all lung US scans in less than one minute, and Cibinel et al. ${ }^{12}$ completed lung US in under five minutes. This is consistent with previous studies. ${ }^{27}$ Thus, lung US may allow for more rapid diagnosis and timely, appropriate management. These 
findings are important for patients as delayed, inaccurate diagnoses have been shown to increase morbidity and mortality. ${ }^{13}$

Given that the use of bedside US is growing rapidly, this review applies to a range of providers and settings. We did not limit our analysis to academic EDs because lung US may be particularly useful in community-based centres where laboratory (e.g., BNP assays) and radiographic testing may not be widely available. Importantly, we included studies with non-expert sonographers to broaden the application of the results. As such, the analysis includes US scans performed by emergency physicians, cardiologists, emergency medicine residents, ultrasound fellows, and medical students (Table 1). Two studies that did not exclusively use physician operators ${ }^{1,10}$ demonstrated high inter-rater reliability while comparing novice sonographers with experts ( $\mathrm{k}=82 \%$ and $92 \%$, respectively). This is likely because of the relative simplicity of image generation from lung US and recognition of B-lines. This may further extend the applicability of this study to physicians who have adequate training in ultrasound but may not be considered lung sonography experts.

In analyzing the results, it is clear that the present study had outliers with respect to both sensitivity and specificity. Chiem et al. ${ }^{10}$ and Liteplo et al. ${ }^{1}$ both reported a sensitivity of $57 \%$ but had specificity values in keeping with the overall meta-analysis (84\% and $85 \%$, respectively). Both of these studies included non-physician sonographers and demonstrated high inter-rater reliability between novices and experts (92\% and 82\%, respectively). Of interest, treatment for $\mathrm{ADHF}$ had been initiated in $36 \%$ of the patients included in the Chiem et al. study, ${ }^{10}$ and Liteplo et al. only enrolled patients if their attending physician had ordered an NT-proBNP assay. ${ }^{1}$ This may have created a selection bias for the inclusion of patients who were more likely to have ADHF. Kajimoto et al. ${ }^{22}$ reported that the diagnostic sensitivity and specificity were 96\% (95\% CI 86.1\%$99.1 \%$ ) and $54.1 \%$ (95\% CI 38.1\%-69.2\%), respectively. As noted in Table 1, the US protocol in this study was performed by a cardiologist and included bedside echocardiography and an IVC measurement (after a lung US examination). The high sensitivity and low specificity in this study were likely secondary to the lung US protocol. This study defined pulmonary edema as the presence of any number of B-lines in two or more zones bilaterally.

Our summary estimates of the sensitivity and specificity for lung US (Figure 2) were lower than those reported in a previous systematic review by $\mathrm{Al}$ Deeb et al., which included both hospitalized and ED patients. ${ }^{5}$ The explanation for the difference is likely multifactorial and includes disease severity, ongoing treatment, and sonographer skill, among other variables potentially unique to the ED. Additionally, in a recent meta-analysis, Martindale et al. ${ }^{18}$ reported a sensitivity of $85 \%$ and specificity of $93 \%$, both of which were higher than our estimates. Their review included three studies that were excluded from our analysis but may have accounted for these differences. Among these, Prosen et al. ${ }^{27}$ (2011) evaluated lung US in prehospital patients and reported a sensitivity of $100 \%$ and specificity of $94 \%$. A 2012 study by Piccoli et al. that examined multiple diagnostic modalities was excluded from our review because the clinical diagnosis was not blinded from the lung US results. ${ }^{28}$ Thus, although our results suggest that B-lines might be less accurate than indicated by previous studies, B-lines may provide a more realistic estimate for early lung US in the ED, as the disease spectrum and severity likely differ from those seen in the ICU and inpatient settings.

Although the summary sensitivity and specificity from our analysis are promising, there was considerable heterogeneity between studies $\left(I^{2}=93.7\right)$ that suggests these results must be interpreted with caution. A subgroup analysis revealed that the sample sizes contributed to the heterogeneity; however, we recognize that there were likely additional factors that were not uncovered in the subgroup analysis including US protocol and patient population, as well as the timing of the lung US. Although all scans were performed early and before the availability of any other diagnostic tests, the time was not recorded in any of the included studies. As mentioned, the studies that included learners represented outliers in the data, and, accordingly, the second meta-analysis that excluded these studies $(N=5)$ reduced the level of heterogeneity $\left(I^{2}=77.2\right)$, while modestly improving the diagnostic accuracy (sensitivity $=88.6 \%$; specificity $=83.2 \%$ ). This improved level of heterogeneity makes the interpretation of the diagnostic accuracy more reliable; however, the sample size for the physician-only analysis was small.

\section{LIMITATIONS}

We recognize there were limitations to this review and its interpretation, perhaps most notably, the heterogeneity among the included studies. The variation in 
sample sizes contributed significantly to the heterogeneity of our meta-analysis. However, there are likely many contributing factors. The seven studies were drawn from four different countries involving a range of hospital sizes. Despite the fact that all scans were performed early in the ED, the timing of the scans was variable. Further, some patients had received their initial treatment for ADHF before scanning. There is currently no gold standard diagnostic test for ADHF; therefore, studies must rely on retrospective chart reviews that are limited to the reviewers' interpretation. We also recognize that variability was inherent in this meta-analysis because bedside lung US is not pathognomonic for ADHF. Previous studies have shown bilateral B-lines in ARDS, multi-lobar pneumonia, and tuberculosis, among others. ${ }^{2,22}$

All studies ensured proper blinding of the sonographer to the diagnostic test results. It is challenging, however, to effectively blind a sonographer to their own gestalt based on a patient's interaction. That is, those performing the US may use their own clinical experience to predict $\mathrm{ADHF}$, while observing the patient during the test. As this may be experience dependant, the effect may be greater for physicians than junior learners. It is also apparent that the level of US training was inconsistent between studies. All included studies required formal training; however, some sonographers had higher levels of training that may have improved their accuracy. Interestingly, Frasure et al. recently suggested that patient positioning may also impact the number of B-lines seen during the US examination of a patient with pulmonary edema. ${ }^{29}$ The studies included in this assessment did not reliably report patient positioning; therefore, we could not account for potential differences.

Concerning our systematic search, the 18 studies included in the full-text review were found to be conference abstracts upon retrieval with no associated full text. The authors were contacted to ensure that the publications were not pending. This suggests there is currently a large number of ongoing relevant studies that may improve upon the aforementioned limitations in the current review and offer further insight into the diagnosis of $\mathrm{ADHF}$ in the $\mathrm{ED}$.

\section{CONCLUSIONS}

This systematic review and meta-analysis focused on the accuracy of B-lines in early bedside lung US for the diagnosis of ADHF in the ED. The included studies suggest that bedside lung US may serve as a reliable diagnostic adjunct for ED patients with undifferentiated dyspnea for whom physicians have a clinical suspicion of ADHF. Based on our meta-analysis, we suggest performing early bedside lung US and considering the initiation of treatment, while awaiting additional test results for dyspneic patients. For the skilled sonographer, we would recommend incorporating bedside echocardiography and IVC measurement to delineate further the cause of pulmonary edema, as this may improve the accuracy of isolated B-lines. Our review of ED lung US adds to a growing body of literature on point-of-care US that is applicable to a wide range of physicians working in both urban and rural centres. We have provided support for a diagnostic adjunct that may improve the accuracy and timeliness of the treatment of $\mathrm{ADHF}$, a common condition that greatly impacts the health care system. Further research is needed to assess the level of training necessitated for accurate lung US, in addition to the evaluation of multiple B-line protocols. Perhaps more importantly, research is needed to assess the impact of bedside lung US on patient outcomes in acute undifferentiated dyspnea.

Competing interests: None declared.

\section{SUPPLEMENTARY MATERIALS}

To view supplementary material for this article, please visit https://doi.org/10.1017/cem.2018.27

\section{REFERENCES}

1. Liteplo AS, Marill KA, Villen T, et al. Emergency thoracic ultrasound in the differentiation of the etiology of shortness of breath (ETUDES): sonographic B-lines and N-terminal pro-brain-type natriuretic peptide in diagnosing congestive heart failure. Acad Emerg Med 2009;16 (3):201-10.

2. Volpicelli G, Cardinale L, Garofalo G, Veltri A. Usefulness of lung ultrasound in the bedside distinction between pulmonary edema and exacerbation of COPD. Emerg Radiol 2008;15(3):145-51.

3. Storrow AB, Jenkins CA, Self WH, et al. The burden of acute heart failure on U.S. emergency departments. $7 A C C$ Heart Fail 2014;2(3):269-77.

4. Collins SP, Storrow AB, Levy PD, et al. Early management of patients with acute heart failure: state of the art and future directions-a consensus document from the SAEM/HFSA acute heart failure working group. Acad Emerg Med 2015;22 (1):94-112. 
5. Milne J, Atkinson P, Bowra J, et al. My patient is short of breath: is the problem lung tissue? Ultrasound 2013;21(2):82-7.

6. Al Deeb M, Barbic S, Featherstone R, Dankoff J, Barbic D. Point-of-care ultrasonography for the diagnosis of acute cardiogenic pulmonary edema in patients presenting with acute dyspnea: a systematic review and meta-analysis. Acad Emerg Med 2014;21(8):843-52.

7. Zeng Q, Jiang S. Update in diagnosis and therapy of coexistent chronic obstructive pulmonary disease and chronic heart failure. 7 Thorac Dis 2012;4(3):310-5.

8. Salpeter SR, Ormiston TM, Salpeter EE. Cardiovascular effects of $\beta$-agonists in patients with asthma and COPD: a meta-analysis. Chest 2004;125(6):2309-21.

9. Wang CS, FitzGerald JM, Schulzer M, Mak E, Ayas NT. Does this dyspneic patient in the emergency department have congestive heart failure? $7 A M A$ 2005;294(15):1944-56.

10. Chiem AT, Chan CH, Ander DS, Kobylivker AN, Manson WC. Comparison of expert and novice sonographers' performance in focused lung ultrasonography in dyspnea (FLUID) to diagnose patients with acute heart failure syndrome. Acad Emerg Med 2015;22(5):564-73.

11. Cardinale L, Priola AM, Moretti F, Volpicelli G. Effectiveness of chest radiography, lung ultrasound and thoracic computed tomography in the diagnosis of congestive heart failure. World 7 Radiol 2014;6(6):230-7.

12. Cibinel GA, Casoli G, Elia F, et al. Diagnostic accuracy and reproducibility of pleural and lung ultrasound in discriminating cardiogenic causes of acute dyspnea in the emergency department. Intern Emerg Med 2012;7(1):65-70.

13. Ray P, Birolleau S, Lefort $Y$, et al. Acute respiratory failure in the elderly: etiology, emergency diagnosis and prognosis. Crit Care 2006;10(3):R82.

14. American College of Emergency Physicians. Emergency ultrasound guidelines. Ann Emerg Med 2009;53(4):550-70.

15. Lichtenstein DA, Mezière GA. Relevance of lung ultrasound in the diagnosis of acute respiratory failure: the BLUE protocol. Chest 2008;134(1):117-25.

16. Volpicelli G, Mussa A, Garofalo G, et al. Bedside lung ultrasound in the assessment of alveolar-interstitial syndrome. Am 7 Emerg Med 2006;24(6):689-96.

17. Gallard E, Redonnet JP, Bourcier JE, et al. Diagnostic performance of cardiopulmonary ultrasound performed by the emergency physician in the management of acute dyspnea. Am $\mathcal{f}$ Emerg Med 2015;33(3):352-8.

18. Martindale JL, Wakai A, Collins SP, et al. Diagnosing acute heart failure in the emergency department: a systematic review and meta-analysis. Acad Emerg Med 2016;23(3): 223-42.

19. Moher D, Liberati A, Tetzlaff J, Altman DG, PRISMA Group. Preferred reporting items for systematic reviews and meta-analyses: the PRISMA statement. BM7 2009: 339. jul21 1:b2535.

20. Critical Appraisal Skills Programme (CASP). CASP Checklists; 2014. Available at: http://media.wix.com/ugd/ dded87_3815f02af1b34c21b8c3b2b5020024c3.pdf.

21. Pivetta E, Goffi A, Lupia E, et al. Lung ultrasoundimplemented diagnosis of acute decompensated heart failure in the ED. Chest 2015;148(1):202-10. http://doi.org/10. 1378/chest.14-2608.

22. Kajimoto K, Madeen K, Nakayama T, et al. Rapid evaluation by lung-cardiac-inferior vena cava (LCI) integrated ultrasound for differentiating heart failure from pulmonary disease as the cause of acute dyspnea in the emergency setting. Cardiovasc Ultrasound 2012;10(1):49.

23. Anderson KL, Jenq KY, Fields JM, Panebianco NL, Dean AJ. Diagnosing heart failure among acutely dyspneic patients with cardiac, inferior vena cava, and lung ultrasonography. Am 7 Emerg Med 2013 Aug; 31(8):1208-14.

24. Fonseca C, Mota T, Morais $\mathrm{H}$, et al. The value of the electrocardiogram and chest X-ray for confirming or refuting a suspected diagnosis of heart failure in the community. Eur 7 Heart Fail 2004;6(6):807-12.

25. Lam LL, Cameron PA, Schneider HG, et al. Meta-analysis: effect of B-type natriuretic peptide testing on clinical outcomes in patients with acute dyspnea in the emergency setting. Ann Intern Med 2010;153(11):728-35.

26. Knudsen CW, Omland T, Clopton P, et al. Diagnostic value of B-Type natriuretic peptide and chest radiographic findings in patients with acute dyspnea. Am $\mathcal{f}$ Med 2004; 116(6):363-8.

27. Prosen G, Klemen P, Štrnad M, Grmec S. Combination of lung ultrasound (a comet-tail sign) and N-terminal probrain natriuretic peptide in differentiating acute heart failure from chronic obstructive pulmonary disease and asthma as cause of acute dyspnea in prehospital emergency setting. Crit Care 2011;15(2):R114.

28. Piccoli A, Codognotto M, Cianci V, et al. Differentiation of cardiac and noncardiac dyspnea using bioelectrical impedance vector analysis (BIVA). 7 Card Fail 2012;18(3):226-32.

29. Frasure SE, Matilsky DK, Siadecki SD, et al. Impact of patient positioning on lung ultrasound findings in acute heart failure. Eur Heart $\mathcal{F}$ Acute Cardiovasc Care 2015; 4(4):326-32. 